\title{
A Heuristic for Approximating Extreme Negative Price Returns in Financial Markets
}

\author{
J.T. MANHIRE ${ }^{a, *}$ \\ ${ }^{a}$ Texas A\&M University, 1249 TAMU, College Station, TX 77843-1249, U.S.A.
}

\begin{abstract}
The paper describes the behavior of financial markets as functions of the variables "price return" and "time" based on the net difference between ask and bid volumes over a unit period, thereby suggesting that at least a negative non-trivial price return extreme exists for a unit period. This admittedly heuristic approach also offers a method for approximating these negative price return extremes for a specific unit period. Limitations and applications are discussed.
\end{abstract}

DOI: 10.12693/APhysPolA.133.1408

PACS/topics: 02.70.-c, 05.10.-a, 89.65.Gh

\section{Introduction}

This paper attempts to describe the behavior of financial markets as a set of functions in terms of price return and time variables based on the net difference between ask and bid volumes over a unit period, thereby suggesting that at least a negative price return extreme exists for a unit period.

Yet, merely demonstrating that extreme values exist is not enough. In the context of financial markets, the extreme measure that investors and market risk managers care about most is usually a negative price return. If the extreme negative price return - measured as a percent decrease over a defined period - is simply unity (i.e., a complete market collapse to zero), then the suggestion that negative price return extremes exist becomes trivial. This paper introduces a heuristic method to determine whether negative price returns over a defined period are constrained by extremes that are non-trivial.

There are three reasons to focus on price return reductions instead of increases. The first is the reason just mentioned; it is the focus of most market risk managers. The second is that price return increases are theoretically infinite while decreases are limited to the displacement between the current price and zero. The third is extant empirical work. Others observe that the cumulative probability distribution of price returns takes the approximate power law form $x^{-\alpha}$, where $\alpha$ is an appropriate parameter for the market and sampling frequency. This empirical work shows that the power law form holds for different financial markets across different sizes and for different periods [1-3]. Placing an upper limit on price returns would mean that this observed power law form could never be satisfied. ${ }^{\dagger}$ Although this paper uses an extreme price return increase variable as part of its mathematical formalism, it is careful to only claim a method for approximating price return reductions.

\footnotetext{
*e-mail: jmanhire@tamu.edu

†I am grateful to an anonymous reviewer for this observation.
}

Further, this method is only potentially useful for markets that are robust and actively traded, and where the mean value of the price return is close to zero. This last requirement implies a form of mean reversion, which remains controversial in the literature $[4,5]$.

Lastly, this heuristic is based on a uniformitarian-like assumption that, to some extent, historical data can be used to predict extreme price returns. There is significant disagreement in the literature over whether this postulate holds [8-11]. Consequently, this underlying assumption might be a significant limitation of the method.

This paper does not use a statistical approach in suggesting that at least extreme negative price returns exist. Instead, it focuses on simple mechanics. The paper does employ some basic probability methods in the third section, but these are transitional in arriving at proposition of non-triviality. The paper's conclusion offers an approximation of the magnitude of a negative price return extreme. In this way, the paper might make some substantive contribution to the literature and market risk management [12-16].

The paper is divided into five sections. The first is this brief introduction. The second defines a financial market as a system dependent entirely upon the interactions of generalized buyers and sellers and then offers an inductive argument that suggests the existence of negative price return extremes. The third section offers a way to approximate these negative price return extremes to determine if they are non-trivial. The fourth discusses limitations of the heuristic and presents an application. The fifth section offers a brief summary.

\section{Does a negative price return extreme exist?}

We begin by defining the variables of a financial market and examining the different perspectives from which one can view these variables as coordinates. We will then derive a general equation of market dynamics based on these perspectives and show that the solution suggests the existence of negative price return extremes.

Define a unit period as $t_{0} \rightarrow t_{1}$ with the quantity $t=$ $t_{1}-t_{0}=1$ unit of elapsed time. Define an asset's price 
as a function of time, or $p(t)$, where the change in price over one unit of elapsed time is $\Delta p(t)=p\left(t_{1}\right)-p\left(t_{0}\right)$. Define $r(t)$ as the "price return" for the unit period $t$ and $|r(t)|$ as the modulus of the price return, where $r(t)=$ $\Delta p(t) / p\left(t_{0}\right)$.

A financial asset has a price return configuration space $V(r)=\left\{r_{j} \in \mathbb{R}: j \in \mathbb{N}\right\}$. An asset also has a set of possible price return configurations as functions of elapsed time $\{r(t) \in \mathbb{R}: t \geq 0, t \in \mathbb{R}\} \subseteq V(r)$. The price return dynamics of a financial market are time-dependent. Thus, a market's price return configuration space is a vector field defined by price return coordinates $r(t)$ that have the time derivatives $\dot{r}(t), \ddot{r}(t)$, etc.

We use the term "price return" here since it is more common in the financial market literature. We use it without regard to dividends because we're only interested in the normalized movement of the price based on the starting price $p\left(t_{0}\right)$, not the "total" return for investment purposes per se..$^{\ddagger}$ Thus, one can think of the price return (as the term is used here) as the physical displacement of $r$ on the vector field $V(r)$.

Because we are primarily concerned with this normalized change in price (price return) for a unit period, we can imagine a "price return-time" coordinate system $K$ such that $t_{0}=r\left(t_{0}\right)=0, t_{1}=t$, and $r\left(t_{1}\right)=r(t)$. Let us arbitrarily, but intuitively, designate an increase in price return as positive $(+)$ and a decrease as negative $(-)$ in $K$. If thought of as a system of rectangular coordinates, we can imagine $t$ along the abscissa and $r(t)$ along the ordinate similar to a typical financial market chart. We will first consider an asset moving up or down along the stationary one-dimensional vector field $V(r)$. Let us call this first frame of reference $K_{1}$.

In an active market, traders with the desire to buy an asset place bid orders and traders wishing to sell place ask orders. Yet, desire is not enough to directly affect a change in the price of an asset. There must also exist an interaction with another trader to turn a bid or ask order into a transaction. We will call transactions associated with bids "bid volume." This becomes the scalar quantity $B$ representing the number of bid transactions for a unit period. "Ask volume" is similarly the scalar quantity $A$ representing the number of ask transactions for a unit period.

Analysis of historical data from active markets suggests $B$ has the potential to reduce prices while $A$ has the potential to increase prices. The bid price is defined as the highest current price at which a trader is willing to buy. The ask price is defined as the lowest current price at which a trader is willing to sell. Therefore, as bid transactions transpire, the once highest bid price vanishes and the next highest (but lower than before) price

$\ddagger$ "Total returns" for investment purposes typically include dividends or other income produced by the asset separate from any capital gain. I am again grateful to an anonymous reviewer for pointing out this distinction. becomes the highest current price. As ask transactions transpire, the once lowest ask price vanishes and the next lowest (but higher than before) price becomes the lowest current price. Thus, $B$ is selling volume and $A$ is buying volume [17].

When a financial market experiences more buying volume than selling volume, there are more traders buying at the ask price. This "pushes" the asset up in price (it changes the price in the positive direction). When a market is experiencing more selling volume than buying volume, there are more traders selling at the bid price, which "pushes" the asset down in price (it changes the price in the negative direction). Because we define the price return $r$ as a function of these positive or negative changes in price, we claim that the net effect (i.e., the result we can observe) of bid volume on the price return $r$ in $K_{1}$ is the product of the bid volume $B$ and the negative price return $-r_{B}$ since we arbitrarily defined price return reductions as negative. Likewise, the net effect of ask volume is the product of the ask volume $A$ and the positive price return $r_{A}$ since we arbitrarily defined price return increases as positive.

From this we see that the net effect of all bid volume for a unit period changes the state of the asset along the stationary price return dimension in the negative direction from $r\left(t_{0}\right)$ to $-r\left(t_{1}\right)$. This negatively-directed result is proportional to the effect of bid volume on the price return attributable to it, or $-\ddot{r}_{B} \propto(B)\left(-r_{B}\right)$. Similarly, we see that the net effect of all ask volume for a unit period changes the state of the asset along the price return dimension in the positive direction from $r\left(t_{0}\right)$ to $+r\left(t_{1}\right)$, or that $\ddot{r}_{A} \propto(A)\left(r_{A}\right)$. If we think classically about these mechanics, we find that they are consistent with the dynamics underlying Hooke's law and, therefore, should not be unfamiliar.

Historical data analysis indicates that not all markets given identical ask and bid volumes have the same ask and bid volume effects with respect to positive and negative changes in price returns. As a result, we can assume that each market additionally has some specific inertial property - let us call it $n$ - that constrains the asset in its positive or negative movement away from $r\left(t_{0}\right)$ in $K$ for a specific unit of time. Essentially, $n$ is some number specific to a particular market for that unit period that is inversely proportional to the change in the price return. The lower the number, the more likely the asset is to move further from $r\left(t_{0}\right)$, and vice versa. Therefore, $n$ is minimized when the modulus $|r(t)|$ is maximized, and $n$ is maximized in the limit as $|r(t)| \rightarrow 0$. As a result, we can express the price effects of bid and ask volumes as ratios specific to each market. We will call these specific ask volumes and specific bid volumes, respectively.

Of course, this description is incomplete. Ask and bid volumes do not exist in isolation. In an active market, there is always a superposition of specific ask and specific bid volumes over any unit period where the asset is pushed in the positive price return direction when the ask volume dominates and pushed in the negative price 
return direction when the bid volume dominates. Recalling the underlying dynamics of Hooke's law mentioned earlier, we see that this specific net volume gives us

$$
\left(\frac{A-B}{n}\right) r(t)=\ddot{r}(t)
$$

where $r(t)=r_{A}-r_{B}$. From this, we can define the net effect on the price return for a unit period (the dominating net "push") as $\ddot{r}(t)=\ddot{r}_{A}-\ddot{r}_{B}$, where $\ddot{r}(t)$ is positive if $A$ dominates for the unit period and negative if $B$ dominates. Thus, the net effect of the specific net volume which we will call $G(r)$ - equals this net "push" and the direction is determined by whether the ask or bid volume dominates for that particular unit period. This yields the expression

$$
G(r)=\ddot{r}(t) .
$$

Again, this is the equation for an asset moving through the stationary price return dimension resulting from the net effect of the specific ask and specific bid volumes for that unit period. It is, in fact, market mechanics as described from the perspective of the vector field $V(r)$.

Now let us look at this behavior from a second frame of reference, $K_{2}$. This new perspective is identical to $K_{1}$ in all respects except one: instead of the price return dimension being stationary and the asset moving along it, in $K_{2}$ the asset is fixed and $V(r)$ moves up and down relative to the stationary asset. This becomes the change in the price return as described from the perspective of the asset. The specific ask and bid volumes remain the cause of any change in $V(r)$ just as in $K_{1}$. The only difference between $K_{1}$ and $K_{2}$ is what we consider to be stationary and what we consider to be changing. Each produces indistinguishable data regarding price return magnitudes and their changes.

After imagining this behavior from the $K_{2}$ perspective, we can conclude that to get the same effect as observed in $K_{1}$ this new frame of reference must experience oppositely-directed changes in $V(r)$. Therefore, every \pm price return change in the asset resulting from the net effect of specific ask and bid volumes in $K_{1}$ is the same as a $\mp$ change in $V(r)$ in $K_{2}$. This means

$$
G(r)=-V^{\prime}(r),
$$

where the prime denotes a price return derivative just as the dot denotes a time derivative. Combining Eqs. (1) and (2) gives us the dynamics of a market in terms of both changes in time and price return, or $\ddot{r}(t)=-V^{\prime}(r)$.

But what does $V^{\prime}(r)$ comprise? Recall that $r\left(t_{0}\right)=0$ in $K$, which we will now express as simply $r_{0}$ for concision. A review of historical data from active, robustlytraded markets reveals that the mean price return for a unit period tends to be relatively close to zero in $K$, the specific frame of reference notwithstanding. Therefore, we can approximate the price return vector field with the following Maclaurin series:

$$
V(r) \approx V\left(r_{0}\right)+\frac{V^{\prime}\left(r_{0}\right)}{n} r+\frac{V^{\prime \prime}\left(r_{0}\right)}{2 n} r^{2}+\ldots
$$

(N.B.: For markets where the mean price return for a unit period is not relatively close to zero in $K$, this assumption cannot hold.)

The first two terms in Eq. (3) give us no new information about $V(r)$. Thus, the first meaningful term in this series is $V^{\prime \prime}\left(r_{0}\right)$. Because changes in the price return derivative of the vector field are uniform for all price return values, we see that $V^{\prime \prime}\left(r_{0}\right)$ is some mean constant of the vector field for that particular unit period $t$. Let us call this mean constant $h$. Therefore, from Eq. (3) we get

$$
V(r) \approx \frac{r^{2} h}{2 n}
$$

Yet, as we just discussed, the only meaningful mean constant for any given unit period is the net (ask and bid) volume for that period since we know from Eqs. (1) and (2) that

$$
-V^{\prime \prime}(r)=G^{\prime}(r)=\frac{A-B}{n}=-H,
$$

where $H$ is the specific net volume $h / n$. Therefore, $H=(B-A) / n$.

From Eq. (4), we see that $V^{\prime}(r) \approx H r(t)$. Consequently, the price return dynamics for any unit period in $K$, from both perspectives, can be expressed as the harmonic approximation

$$
G(r)+H r(t)=0 .
$$

This is consistent with Eq. (2).

Equation (5) asks us for a solution to the second-order linear ordinary differential equation $\frac{d^{2}}{d t^{2}} r(t)+H r(t)=0$. Assume the solution is proportional to $\exp (\beta t)$ where $\beta$ is a constant. This gives us $\beta^{2} \exp (\beta t)+H \exp (\beta t)=0$. For a finite $\beta, \exp (\beta t) \neq 0$. This leaves the roots of the polynomial $\beta^{2}+H=0$, which are $\beta=i \sqrt{H}$ and $\beta=-i \sqrt{H}$, where $i$ is the imaginary unit. This yields

$$
\begin{aligned}
& r_{1}(t)=u \exp \left(\varphi_{0}+t \sqrt{\frac{A-B}{n}}\right), \\
& r_{2}(t)=v \exp \left(\varphi_{0}-t \sqrt{\frac{A-B}{n}}\right),
\end{aligned}
$$

where $u$ and $v$ are arbitrary constants and $\varphi_{0}$ is the initial condition of the phase $\varphi$ in $K$ (we will define and examine $\varphi$ more closely in just a minute).

We hold that $r(t)=r_{1}(t)+r_{2}(t)$ is the projection of the complex number $z$ and its complex conjugate $z^{*}$ on the real axis. If we assume that $u=v=|z| / 2$, we get the following well-known solution to Eq. (5):

$$
r(t)=\frac{1}{2}\left(z+z^{*}\right)=\frac{1}{2}\left(|z| \mathrm{e}^{\mathrm{i} \varphi}+|z| \mathrm{e}^{-\mathrm{i} \varphi}\right)=|z| \cos \varphi \cdot(6)
$$

Here, the phase, or principal argument of $z$, is

$$
\varphi=\operatorname{Arg}(z)=\dot{\varphi} t+\varphi_{0} .
$$

The change in the phase for each unit period is the radial frequency $\dot{\varphi}$, and the square of this time derivative is $H$. 
Thus, for a unit period, $\dot{\varphi}(t)=\varphi-\varphi_{0}$. If $\varphi_{0}$ is constant, it is clear that one can determine $r(t)$ from $\dot{\varphi}(t)$.

In solving Eq. (5) as an initial value problem, we see that $\varphi(t)=\int \sqrt{H} d t=t \sqrt{H}+C$. Here we interpret the constant of integration as the starting point of $\varphi$ in $K$ at $t_{0}$, or $C=\varphi_{0}$, which is consistent with Eq. (7). This means $\varphi_{0}$ is constant in $K$ so one can determine $r(t)$ for a specific unit period from $\dot{\varphi}(t)$. Therefore, we see from Eq. (6) that the price return is a function of the net ask and bid volumes over a unit period. This is consistent with our initial assumption that any change in the price return is a direct result of the total ask and bid transactions for a unit period. There are no other factors directly affecting the mechanics of $r(t)$.

Additionally, $|z|$ is the complex modulus in Eq. (6). We can regard this also as a radius vector on the complex plane. Thus, Eq. (6) becomes

$$
r(t)=|z| \cos \varphi \text {. }
$$

The cosine function, by definition, has the property $\cos \varphi \geq-1$. Multiplying through by $|z|$ gives us $r(t) \geq$ $-|z|$. Consequently, the negative complex modulus is equivalent to the negative extreme price return magnitude. We can conclude, therefore, that for a given unit period there exists at least a negative extreme price return of $r(t)=-|z|$.

There are two conditions to this conclusion. The first is that a market might never reach $-|z|$ in the unit period $t$, so it might never be observed. We claim only that at least the negative extreme theoretically exists for any unit period $t$.

The second condition stresses the final prepositional phrase of the previous sentence: the measure of a price return extreme is unique only for a specific unit period $t$. The distinction here is that we conclude a negative extreme price return theoretically exists for any specific unit period; not for a specific market over a sequence of the same unit periods. In this way, the conclusion here does not violate the empirical power law discussed in the first section.

\section{Are negative price return extremes non-trivial?}

Now that we have provided evidence that negative price return extremes might exist, the next step is to decide under what conditions these extremes approach triviality, if any. Define triviality as an extreme price return $r(t)=-1$. In other words, even if price return extremes exist, they become trivial if all we can say about them is that a market will hit a zero-price floor for some unit period. The conclusion that a market losing all value can be defined as an extreme negative price return is selfevident and, therefore, trivial. Hence, the only price return extremes that are meaningful (non-trivial) to any risk analysis are those between -1 and 0 . As previously discussed, the price return might exceed unity in the positive direction, but never in the negative direction.
Here we introduce the Lagrangian function $L(r, \dot{r}, t)$, which is a function of the price return coordinates, their time derivatives, and time. It contains the same information about the dynamics of a market in $K$ as contained by Eq. (5). $\$$ From the time integral of the Lagrangian of Eq. (5) we get the following action functional of an asset:

$$
\begin{aligned}
& S[r(t)]= \\
& \int_{t_{0}}^{t_{1}} \mathrm{~d} t\left[L(r, \dot{r}, t)=\frac{1}{2}\left(\frac{A-B}{n} r^{2}+\dot{r}^{2}\right)\right]=\frac{n r^{2}}{2 t} .
\end{aligned}
$$

We see that $\sqrt{S}=+r \sqrt{n / 2}$ and $-\sqrt{S}=-r \sqrt{n / 2}$ since $t=1$ for any unit period. This means that besides the price return, the remainder of the action functional can be regarded as essentially a constant due to the inverse square relationship between $n$ and $r$. As a result, $\operatorname{Pr}( \pm r) \equiv \operatorname{Pr}( \pm r \sqrt{n / 2})$ for any given market.

For the probability that either the positive or negative square root of the action functional is on the interval between the extreme values of the square root of the action functional, we can write

$$
\begin{aligned}
& \operatorname{Pr}(-|z| \sqrt{n / 2} \leq[(-\sqrt{S}) \text { or }(+\sqrt{S})] \leq+|z| \sqrt{n / 2}) \\
& =1-\operatorname{erfc}(|z| \sqrt{n / 2})^{2},
\end{aligned}
$$

where $\operatorname{erfc}(X)$ is the complementary Gauss error function, defined as $1-\operatorname{Pr}(-X \leq x \leq X)$. Recall that $n$ is a minimum at this extreme.

Because $|z|$ is an extreme value, this probability must approach certainty since by definition all measures for the positive or negative square root of the action functional must fall between the positive or negative square root of the action functionals containing the positive or negative extreme price returns. We can include the positive values here since they are at least as extreme as the negative values, although we do not contend that an upper limit exists for the reasons we explained previously. This means that $\operatorname{erfc}(|z| \sqrt{n / 2})^{2} \rightarrow 0$.

We can define the phase $\varphi$ as having values on the interval between $\pm \pi$, where $\pi$ is a maximum radial measure in the complex plane that is consistent with both the generally-accepted range of the principal value of $\operatorname{Arg}(z)[18]$ and the fact that cosine is an even function in Eq. (6). As with the probability of the square roots of the action functional, we see that $1-\operatorname{erfc}(\pi)^{2} \rightarrow 1$, which implies $\operatorname{erfc}(\pi)^{2} \rightarrow 0$. Therefore,

$$
\operatorname{erfc}(|z| \sqrt{n / 2})^{2}=\operatorname{erfc}(\pi)^{2} \text {. }
$$

This is only an approximation, but we assume the approximation close enough to express the relationship as an equality for our heuristic purposes.

\footnotetext{
$\S$ The Lagrangian of a market actually precedes its equation of motion; i.e., we construct Eq. (5) from the Lagrangian in Eq. (8).
} 
Another way to think about this is to return to the complex plane from the previous section. If the real price return $r(t)$ is the median of $z(t)$ and $z^{*}(t)$, then there are always two complex numbers and two phases for each real price return on $K$. For example, at $t_{0}$ of any unit period $\varphi_{0}=\frac{\pi}{2}$ in complex vector space, but there is also a phase in the complex conjugate (dual) vector space where $\varphi_{0}^{*}=-\frac{\pi}{2}$. Similarly, there is a number $z$ in complex space for every real price return as well as a number $z^{*}$ in the complex conjugate space [19]. Therefore, the probability of any $r(t)$ is equivalent to the probability of $z$ and the probability of $z^{*}$, i.e., $\operatorname{Pr}(r) \equiv \operatorname{Pr}(z) \operatorname{Pr}\left(z^{*}\right) \equiv \operatorname{Pr}(|z|)^{2}$. It is also equivalent to the probability of $\varphi$ and the probability of $\varphi^{*}$, i.e., $\operatorname{Pr}(r) \equiv \operatorname{Pr}(\varphi) \operatorname{Pr}\left(\varphi^{*}\right) \equiv \operatorname{Pr}(|\varphi|)^{2}$.

If we take the inverse complementary error function of both sides of Eq. (9) we get $|z| \sqrt{n / 2}=\pi$, which we can express as

$$
|z|^{2} n=\gamma
$$

if we define the constant $\gamma:=2 \pi^{2}$.

Equation (10) still requires knowledge of $n$ for each market to find the value of $|z|$. Since $n$ is not a number regularly measured, we should try to approximate $|z|$ without relying on a known minimized value of $n$. If we look at the relationship between the absolute value of a specific price return value $\rho$ (i.e., $\rho \geq 0$ ) of a market and the probability that other values of $|r(t)|$ in the same market are greater than $\rho$, we conjecture that

$$
\operatorname{Pr}(|r(t)|>\rho)=\operatorname{erfc}\left(\rho \sqrt{\frac{n}{2}}\right)^{2}=\exp \left(-\frac{\rho}{\mu}\right),
$$

where $\mu$ is the expectation value of $|r(t)|$. From this conjecture, we assume the following:

1. The measure of centrality (expectation value) of $|r(t)|$ is the arithmetic mean of the absolute value of all observed price returns for some order of $t$;

2. The measure of centrality of the probability that any $|r(t)|$ is greater than a specific $\rho$ is the geometric mean of all possible probabilities; i.e., all real numbers on the interval $[0,1]$; and

\section{These measures of centrality coincide.}

From these three assumptions, we can define $\mu$ as the specific price return that maps to the probability of any $|r(t)|$ being greater than the specific absolute price return $\rho$ that is equal to $1 / \mathrm{e}$, or

$$
\mu:=\rho \mapsto \operatorname{Pr}(|r(t)|>\rho)=\exp (-1),
$$

since the geometric mean of all real numbers on the interval $[0,1]$ is $\exp (-1)$. This is a special case of Eq. (11) when $\mu=\rho$.

We can next substitute $|z|$ for $\rho$ in Eq. (11) to get

$$
\operatorname{erfc}\left(|z| \sqrt{\frac{n}{2}}\right)^{2}=\exp \left(-\frac{|z|}{\mu}\right) .
$$

From this and Eq. (9), we see that

$$
\operatorname{erfc}(\pi)^{2}=\exp \left(-\frac{|z|}{\mu}\right) .
$$

If we define the constant $\lambda:=\log \left(\operatorname{erfc}(\pi)^{2}\right)$, this equation becomes

$$
-|z|=\mu \lambda \text {. }
$$

Thus, we find that a market's extreme negative price return is linearly dependent on the expectation value of its absolute price return.

Because both $\gamma$ and $\lambda$ are constants relating to the radial measure of the phase, we can combine them into a single constant $k=\gamma / \lambda^{2}$. From Eqs. (10) and (14), we can then approximate each market's unique minimum inertial constraint at its negative price return extreme with the expression $n=k / \mu^{2}$ since $\mu$ is calculable from historical data.

\section{Limitations}

The method outlined here is not without limitations, the first of which is that this method is merely a heuristic and not a complete theory. It plays with the idea of market movements using the more rudimentary tools of classical mechanics in an attempt to approximate negative price return extremes for any given period. It does not pretend to be, nor should it be taken as, anything more.

As mentioned previously, this method is only potentially useful for markets that are robust and actively traded. It cannot be applied to markets where the mean of the price return over a sequence of unit periods is not close to zero. This requirement implies a form of mean reversion, which remains controversial in the literature.

The heuristic also suffers from an asymmetry in that it only approximates lower limits of price returns and intentionally fails to acknowledge an upper limit. This allows the heuristic to escape conflict with the power law form of a probability distribution function observed empirically, but it also suggests that the magnitude of the radial vector $|z|$ is potentially different for positive price returns than for negative price returns for a specific unit period. On the complex plane, this presents as an extreme negative price return of $-r(t)=-|z|$, but as an extreme positive price return of $r(t)=\infty$. While this is possible, it is certainly odd and is perhaps evidence that the heuristic is incomplete in its understanding of market mechanics.

Another significant limitation of this heuristic is that it relies heavily on the sample with which one chooses to calculate $\mu$ since, per Eq. (14), the accuracy of one's approximation of $-|z|$ depends wholly on this sample. Therefore, this method cannot claim to approximate fixed extreme price return measures for each market since some degree of selection bias in a choice of sample for $\mu$ is unavoidable [20]. While it is possible with this heuristic to approximate an extreme negative price return given sufficient historical data, any such approximation is only valid up to the time of the analysis itself and the sample period chosen to calculate $\mu$. The extreme measure will 
most likely vary over time for each market and should not be considered an absolute negative price return extreme for the sequence of all unit periods. In other words, the inertial coefficient $n$ remains in flux for each market.

Additionally, this heuristic is based on a uniformitarian-like assumption that, to some extent, historical data can be used to predict extreme price returns. This is, again, because the approximation of $-|z|$ depends entirely on the the sample chosen to calculate $\mu$. There is significant disagreement in the literature over whether historical data can be used predictively.

Still, this method might improve our understanding of the rules that undergird market mechanics and, therefore, serve as an additional tool for managing market risk when faced with negative price returns that deviate significantly from historic expectations. For example, based on historical data prior to the market close of October 16, 1987, Eq. (14) would have approximated a daily negative price return extreme for Coca-Cola Company stock (symbol: KO) of $-|z|=-0.2661$ for $t=1$ trading day. During the next trading day, October 19, 1987, KO reached an intraday low ratio of -0.2846 , but closed at $r(t)=-0.2451$. The approximation of $-|z|=-0.2661$ would have been of some assurance for KO's risk managers (and a potential buying opportunity for traders) at $-0.2846 \leq r(t)<-|z|$. More recently, there were similar assurances/opportunities for Automated Data Processing, Inc. (ADP) on May 6, 2010, and Bitcoin (BTC/USD) on August 1, 2014, to name a few.

\section{Summary}

By regarding market mechanics in terms of time and price returns from two separate perspectives, we have presented a heuristic suggesting that financial markets might have extreme negative price returns for specific unit periods. These extremes appear non-trivial except in the limits as $\mu \rightarrow 1 / \lambda$ or as $n \rightarrow \gamma^{+}$. Historical data analysis can illumine whether such non-triviality is common in active markets.

\section{Acknowledgments}

I wish to express my gratitude to Christopher L. Culp, Nuno Garoupa, Joseph R. Hanley, James McGrath, Ann M. Manhire, Andrew P. Morriss, Lisa A. Rich, Saurabh Vishnubhakat, and an anonymous referee for their valuable suggestions. All errors are mine alone.

\section{References}

[1] X. Gabaix, P. Gopikrishnan, V. Plerou, H.E. Stanley, Nature 423, 267 (2003).

[2] V. Plerou, P. Gopikrishnan, L.A.N. Amaral, M. Meyer, H.E. Stanley, Phys. Rev. E 60, 6519 (1999).

[3] P. Gopikrishnan, V. Plerou, L.A.N. Amaral, M. Meyer, H.E. Stanley, Phys. Rev. E 60, 5305 (1999).

[4] J.M. Poterba, L.H. Summers, J. Financ. Econom. 22, 27 (1988).

[5] M.J. Kim, C.R. Nelson, R. Startz, Rev. Econom. Stud. 58, 515 (1991).

[6] R. Balvers, Y. Wu, E. Gilliland, J. Finance 55, 745 (2000).

[7] Y.D. Akarim, S. Sevim, Econom. Modell. 31, 453 (2013).

[8] N. Caldararo, Forum Soc. Econom. 38, 31 (2009).

[9] K. Kohara, T. Ishikawa, Y. Fukuhara, Y. Nakamura, Int. J. Intell. Syst. Acc. Fin. Mgmt. 6, 11 (1997).

[10] S. Jeon, B. Hong, V. Chang, Future Gener. Comput. Syst. 80, 171 (2018).

[11] B.B. Nair, P.K. Saravana Kumar, N.R. Sakthivel, U. Vipin, Expert Syst. Appl. 70, 20 (2017).

[12] C.L. Culp, in: Alternative (Re)Insurance Strategies,Ed. M. Lane, 2nd ed., Risk Books, London 2012, p. 359 .

[13] P. Embrechts, S.I. Resnick, G. Samorodnitsky, North Am. Actuar. J. 3, 30 (1999).

[14] M. Gilli, E. Kellezi, Computat. Econom. 27, 1 (2006).

[15] A.K. Singh, D.E. Allen, P.J. Robert, Math. Comput. Simulat. 94, 310 (2013).

[16] C. Omari, P. Mwita, A. Waititu, J. Math. Finance 7, 846 (2017).

[17] L. Bauwens, P. Giot, Econometric Modelling of Stock Market Intraday Activity, Springer, Boston (MA) 2001, p. 46.

[18] H.S. Kasana, Complex Variables: Theory and Applications, 2nd ed., Prentice Hall-India, New Delhi 2005, p. 14.

[19] K. Schmüdgen, Unbounded Operator Algebras and Representation Theory, Birkhäuser, Basel 1990, p. 16.

[20] A.M. Taylor, Econometrica 69, 473 (2001). 